\title{
Trabajo por proyectos para el desarrollo de competencias en lengua y literatura
}

\author{
(Learning Based Projects to Develope Competencies in Language and \\ Literature)
}

\author{
NÉlida Villafuerte Cosme ${ }^{(\mathbb{D}}$, Centro Universitario del Sur de la Universidad de \\ Guadalajara, Jalisco, México
}

\section{Volumen 2, número 1}

Junio 2017

p. $160-188$

Este número se publicó el 30 de junio de 2017

Artículo recibido: 16 de febrero de 2017

Artículo enviado a corrección: 7 de abril de 2017

Aprobado: 3 de mayo de 2017

ISSN: 2448-5942, doi: https://doi.org/10.36799/el.v2i1.50

\section{Cómo citar}

Villafuerte Cosme, N. (2017). Trabajo por proyectos para el desarrollo de competencias en lengua y literatura. Estudios $\lambda$ ambda. Teoría y práctica de la didáctica en lengua y literatura. , 2(1), 160-188. https://doi.org/10.36799/el.v2i1.50

Derechos de autor: El autor o autores conservan en todo momento sus derechos morales y patrimoniales sobre la obra; la obra no se puede alterar, transformar o ampliar; siempre debe reconocerse la autoría del documento referido. Ninguna de las modalidades de los documentos publicados en Estudios Aambda. Teoría y práctica de la didáctica en lengua y literatura tienen fines comerciales de naturaleza alguna.

Los contenidos de este artículo están bajo una licencia de Creative Commons Atribución No Comercial- Sin

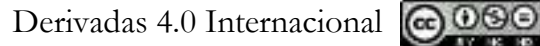




\title{
Trabajo por proyectos para el desarrollo de competencias en lengua y literatura Learning Based Projects to Develop Competencies in Language and Literature
}

\author{
NÉLIDA ViLLAFUERTE COSME ${ }^{1}$
}

\section{RESUMEN}

El trabajo en el aula para desarrollar y aplicar modelos y metodologías centradas en el estudiante es una oportunidad para atender la problemática que representa la comprensión y producción de textos; la educación superior supone que estas capacidades han sido desarrolladas en la trayectoria que corresponde a los niveles básico y medio superior; sin embargo aún en el estudio de licenciatura los alumnos presentan serias debilidades y deficiencias. Por ello se propone un trabajo sobre las estrategias didácticas, el objetivo es mejorar la adquisición de saberes teóricos y prácticos sobre la lengua y la literatura. La propuesta consiste en implementar el trabajo por proyectos como estrategia didáctica para el desarrollo de competencias en estudiantes de educación superior. Esta actividad se ha realizado en la licenciatura de Letras Hispánicas de la Universidad de Guadalajara en el Centro Universitario del Sur. La experiencia didáctica se apoya en una investigación cualitativa cuyos resultados preliminares son: desarrollo de habilidades de investigación, trabajo autogestivo, por objetivos y desarrollo del espíritu emprendedor que se traducen en logros sobre la comprensión de la literatura y la producción de textos, vinculados con una interacción entre los objetos de estudio, su contexto y sus saberes previos, que integran el uso de las tecnologías.

PALABRAS CLAVE: desarrollo de competencias, lectura, escritura, aprendizaje basado en problemas

\begin{abstract}
Classroom work destined to develop and apply models and methodologies centered on the student, is an opportunity to attend to the problems that represent the understanding and production of texts; Higher education assumes that these capacities have been developed in the trajectory that corresponds to the basic and upper middle levels. However even in the undergraduate study students present serious weaknesses and deficiencies. Therefore, we propose a work on didactic strategies, whose objective is to improve the acquisition of theoretical and practical knowledge on language and literature. The proposal, then, is to implement Learning Based Projects (LBP) as a didactic strategy for the development of competences in students of higher education. This activity has been taking place with students of Hispanic Letters in the University of Guadalajara in the Centro Universitario del Sur. This didactic experience is a qualitative research whose preliminary results are the development of research skills, self-management work, learning objectives and the development of the entrepreneurial spirit. All these results translate themselves into achievements on the understanding of literature and the production of texts, linked to an interaction between the objects of study, their context and their previous knowledge, which integrate the use of technologies.
\end{abstract}

KEY WORDS: Competence development, reading, writing, learning based projects

\footnotetext{
${ }^{1}$ Doctora en desarrollo de competencias educativas por el Instituto Pedagógico de Estudios Superiores de Jalisco (IPESUJ), docente de asignatura del Centro Universitario del Sur de la Universidad de Guadalajara, Ciudad Guzmán, Jalisco, México. Miembro de la Benemérita Sociedad de Geografía y Estadística del Estado de Jalisco. Correo: nelidavc@hotmail.com
}

Artículo recibido: 16 de febrero de 2017

Artículo enviado a corrección: 7 de abril de 2017

Aprobado: 3 de mayo de 2017

Villafuerte Cosme doi: https://doi.org/10.36799/el.v2i1.50 Volumen 2, número 1, Año 2017, ISSN: 2448-5942 
"El único hombre que está educado es aquel que ha aprendido cómo aprender; el hombre que ha aprendido como adaptarse al cambio; el hombre que ha aprendido que ningún conocimiento es seguro, que solamente el proceso de buscar conocimiento proporciona bases para la seguridad... El continuo cambio, la confianza en el proceso, más que en los conocimientos estáticos es lo único que tiene sentido como meta de la educación en el mundo moderno... El aprendizaje socialmente más útil en el mundo moderno es aprender el proceso de aprender, una apertura continua a la experiencia y la incorporación en sí mismo del proceso de cambio"

Carl Rogers

\section{INTRODUCCIÓN}

La era 2.0 ha obligado a los estudiantes a modificar su relación con los textos. "podemos desarrollar a la vez muchas estrategias de la competencia comunicativa digital” (Pujolà, 2011, 9), ya que "la computadora forma parte activa en la vida escolar desde el nivel preescolar, por lo que en los niveles medio superior y superior es casi una obligación saber manejarla adecuadamente" (López, 2007, 69); esta tendencia hace presente la idea de Kress que señala que "ahora es imposible dar sentido a los textos, a partir de sus partes lingüísticas solas, sin tener una idea clara de lo que estas otras características [refiriéndose a los elementos externos al texto] podrían estar contribuyendo al significado... De hecho, ya no es posible entender la lengua y sus usos sin entender el efecto de todos los modos de la comunicación que están copresentes en cualquier texto" (Kress, s.f., 337).

"El correo electrónico ha permitido agilizar la comunicación y obtener una inmediatez que hace unos años parecía imposible... internet proporciona un espacio de flujo informativo que ofrece a estudiantes y profesores la posibilidad de acceder a los conocimientos más actuales en sus áreas de formación” (López, 2007, 69); con este marco de referencia pensar en leer y producir textos tiene una nueva dimensión. Lo cual implica un nuevo compromiso de los interesados en desarrollar competencias referentes a la lengua y la literatura.

Para los alumnos del centro universitario del sur de la Universidad de Guadalajara, en la licenciatura de letras hispánicas los retos están definidos en su perfil de egreso (CUSUR, 2008):

Villafuerte Cosme doi: https://doi.org/10.36799/el.v2i1.50 Volumen 2, número 1, Año 2017, ISSN: $2448-5942$ 
Conocimiento profundo en la lengua española en sus estructuras y funciones consideradas tanto sincrónica como diacrónicamente, con especial énfasis en su variante mexicana (conocimiento preceptivo y descriptivo).

$>$ Conocimiento científico en la lengua española en sus manifestaciones literarias, con especial énfasis en sus variantes hispanoamericana y mexicana.

$>$ Acervo intelectual de herramientas teórico-metodológicas para realizar investigaciones lingüísticas y literarias.

$>$ Capacidad tanto de análisis y valoración, como de creación de textos literarios, académicos y otros.

$>$ Capacidad docente en las áreas de la lengua y literatura a nivel medio, mediosuperior y superior, principalmente.

$>$ Capacidad para el diseño, producción y edición de textos.

$>$ Conocimiento de los géneros periodísticos y capacidad para su desempeño.

$>$ Capacidad de documentar y dar testimonio de los fenómenos sociales y culturales, ya sea como cronista o promotor cultural de su localidad.

Estos rasgos orientadores de los contenidos de las unidades de aprendizaje toman forma en la trayectoria curricular, que se desarrolla bajo el enfoque de competencias desde la interpretación de los programas de estudio en concordancia con las diferentes propuestas que abordan este paradigma (ANUIES, 1999; Bravo, 2006; Cortés, 2013; Gil, s.f.; Mir, 2007; Moncada, 2011); alineados desde su fundamentación (UNESCO, 2010 y UNESCO, 2015) y con los acuerdos y avances de la macroeducación (Deusto, 2015; Didrikson, 2000; Latorre, s.f.; Mir, 2007; Rectoría General, 2007; Rodríguez, 2007; Tuning, 2007; Tuning, 2013; Villa y Villa, 2007) interpretados en el aprendizaje centrado en el estudiante, para el caso de esta propuesta, a través del trabajo por proyectos (CIEP, 2015; González y Ramírez, 2011; ITESM, s.f.; Tippelt y Lindemann, 2011).

La idea de aprovechar esta estrategia didáctica es resultado del análisis de la trascendencia de encontrar una forma de compartir experiencias de aprendizaje de acuerdo con la etapa formativa y su proceso de desarrollo de saberes, habilidades y destrezas, ya que el mayor número de estudios se refiere a la educación básica y los esfuerzos de la educación media superior, con las complicaciones de complejidad que representa ( Frade; 2009; Flores; 2010); entre ellas, encontrar una didáctica pertinente en la educación universitaria (Herrán, s.f.; Moreno, s.f.; Nerici, 1973; Programa de Formación de Académicos, 2012; Rodríguez; 2007; Rosell y Más, 2003; Valenzuela, 2004) y su consecuente enfoque para construir secuencias exitosas, (Díaz Barriga, 
2013) reforzadas "por la evidencia de aprendizaje a través de productos observables" (Villafuerte, 2016, 149).

A través de la observación de la práctica docente se conformó esta investigación que ha sido un trabajo transversal de siete años. El inicio fue la primera vez que se impartió la unidad de aprendizaje Ética y gestión de la práctica profesional (en el periodo de agosto del 2009 a enero del 2010), cuyo propósito es la formación de estudiantes de sexto semestre en competencias deontológicas y de propuestas para ejercer la práctica profesional. El programa contemplaba que los estudiantes generaran un proyecto editorial, de gestión cultural o de producción literaria contando con insumos de administración de proyectos y libertad creativa; posteriormente se consideró la transversalidad de estas competencias con la unidad Desarrollo de ambientes de aprendizaje que tenía como base el diseño de actividades de una clase de lengua y literatura, finalmente se ha integrado para el logro de proyectos la unidad Autogestión de proyectos lingüísticos y literarios y aunque no son correspondientes ni comparten prerrequisitos, pueden complementarse y lograr resultados que mejoran las habilidades de lectura y escritura.

En su formación literaria los alumnos han aprendido sobre literatura grecolatina, europea y española (medieval y renacentista), poesía y poética, análisis del discurso, introducción a los estudios literarios, metodología de la investigación literaria (CUSUR, 2008) y después del sexto semestre complementarán sus estudios con literatura hispanoamericana, europea y española hasta el siglo XX y literatura jalisciense; junto con las unidades de aprendizaje de metodologías, didáctica, difusión, creación y estudios lingüísticos, son la gama de oportunidades para leer, escribir, argumentar y pensar multimodalmente cumpliendo con "hacer posible que los ciudadanos sigan aprendiendo durante todo su desarrollo personal [lo que] implica que durante la etapa obligatoria se les haya enseñado a aprender a aprender” (Martín Ortega, 2008 ,72 ). El trabajo por proyectos da esta posibilidad ya que dinamiza la actitud de los estudiantes y permiten hacer nuevas lecturas en la multimodalidad, representación y nueva interpretación de los medios digitales (Kress, 2004, 110-119). Lo cual genera una interacción didáctica multidireccionada y autorregulada por quienes proponen cada proyecto.

En este artículo se reportan los resultados obtenidos al dar seguimiento a un promedio de 18 alumnos por año, donde ha dependido de la decisión de los estudiantes el avance de los proyectos; es decir, las unidades de aprendizaje mencionadas son independientes y al no existir

Villafuerte Cosme doi: https://doi.org/10.36799/el.v2i1.50 Volumen 2, número 1, Año 2017, ISSN: 2448-5942 
prerrequisitos ni correquisitos, la flexibilidad de integrar los créditos de acuerdo al interés de los alumnos hace que no sea una trayectoria lineal, algunos toman al mismo tiempo desarrollo de ambientes de aprendizaje y ética y gestión de la práctica profesional, incluso antes de haber cursado autogestión de proyectos lingüísticos y literarios. Esta es la complejidad de organizar las estrategias didácticas puesto que permanentemente son referentes distintos y niveles heterogéneos de aprendizaje.Ante esta dificultad se han considerado algunos casos representativos de la trayectoria que propone el trabajo por proyectos que es evidencia de logros de desarrollo de competencias no solo de lengua y literatura, también de las que son denominadas como genéricas (Villa y Villa, 2007).

\section{MARCO TEÓRICO}

"En la actualidad, enseñar se hace cada vez más complejo y aprender se ha convertido en una experiencia mucho más desafiante... cada nivel educativo tiene su propia especificidad... y los profesores planifican, organizan, gestionan e implementan el proceso de enseñanzaaprendizaje"

Tiburcio Moreno Olivos

Para comenzar este apartado es preciso señalar que si se considera al proceso de aprendizaje como un sistema y ese mismo sentido se le da al Aprendizaje Basado en Proyectos $(\mathrm{ABP})$, se puede comprender la pertinencia de incidir en las estrategias didácticas como un factor que modifica todo el proceso y que utiliza las características que todos los sistemas comparten: en principio cada sistema existe dentro de otro más grande; por lo tanto estos sistemas son abiertos pues cada uno recibe y proporciona algo a los sistemas contiguos. Los sistemas abiertos se caracterizan por un proceso de cambio infinito con su entorno, cuando el intercambio cesa, el sistema se desintegra porque pierde sus fuentes de energía. Y, por último: "las funciones de un sistema dependen de su estructura" (Bertalanffy, 2006, 100).

El uso de la Teoría de Sistemas en el ámbito educativo es considerado por Gonzalo Gómez Dacal (s.f.), quien señala en su texto La teoria general de sistemas aplicada al análisis del centro escolar una reflexión sobre el uso de este enfoque, con referencia la administración que se utiliza para interpretar los diferentes ámbitos de los centros escolares. Precisa que es común:

Villafuerte Cosme doi: https://doi.org/10.36799/el.v2i1.50 Volumen 2, número 1, Año 2017, ISSN: 2448-5942 
...encontrar la terminología y los principios de la teoría de sistemas en la fijación de objetivos, toma de decisiones, establecimiento de estrategias organizativas, diseño del sistema de comunicación e información, procedimientos de control, etc... Ahora bien, conseguir que una organización funcione, y que funcione con eficacia, exige conocer a la propia organización, a través de sus elementos y de las relaciones de ellos con el todo, a lo que puede contribuir, decisivamente la teoría de sistemas (Gómez, s.f., 5-6).

Este estudio observa a la institución educativa como sistema, para la intervención y la formación de orientadores (Rodríguez, 2007); Valenzuela (2004) describe la aplicación de la misma, considerando a la institución como el macrosistema y al aula como microsistema. En esta perspectiva el modelo integrado de curriculum, la educación basada en competencias, la interacción, la clase, la enseñanza programada, la evaluación, el espacio y el tiempo escolar, son una unidad de análisis. Para este mismo autor el aprendizaje significativo y la metacognición son procesos de autoinstrucción, lo cual implica sistematizar el conocimiento.

Bertalanffy (2006) nos lleva a "través de la teoría que hoy nos permite mirar los productos de las distintas actividades del hombre como partes de una totalidad sistémica integrada y recíproca". Su utilización impactó el área de la administración de las empresas, que consideró el funcionamiento de las organizaciones a partir de la interrelación de sus partes, determinó la estructura autónoma, que incluía la capacidad de reproducirse y conformando la visión de "sistema de sistemas" uno dentro de otro (sector, industria, ambiente). De acuerdo con el pensamiento sistémico, todo es complejo. Cualquier situación tiene un sinnúmero de causas y produce innumerables efectos (Villafuerte, 2016).

Por ello cuando se trata de hablar de desarrollo de competencias se requiere identificar que cada una de ellas funciona como un sistema que se enlaza en el suprasistema de educación superior y a la vez depende de los subsistemas que pueden incluir, gustos, intereses, prioridades, entre otro sin fin de posibilidades. Lo mismo sucede en cuanto a la sistematización del conocimiento que de acuerdo con Rosell y Más (2003) requiere tres reglas:

1) determinar los aspectos o principios según los cuales ha de efectuarse la sistematización,

2) sistematizar todos los objetos aislados $y$

Villafuerte Cosme doi: https://doi.org/10.36799/el.v2i1.50 Volumen 2, número 1, Año 2017, ISSN: 2448-5942 
3) la sistematización solo tendrá éxito y será correcta cuando se lleve a cabo basándose en un solo rasgo o principio determinado.

Estas precisiones validan la importancia de considerar al desarrollo de competencias como un sistema abierto que se modifica de manera compleja. Moreno $(2011,35)$ usa el refrán que reza "Nada es más práctico que una buena teoría" para señalar el valor de la Didáctica y su objeto de estudio, con su trascendencia humana y social; de donde se desprende la idea de implementar el trabajo por proyectos como estrategia didáctica para el desarrollo de competencias, como parte del entramado de la enseñanza, que considera algunos retos: las aulas su espacio y población; la diversidad del alumnado, el curriculum universitario fragmentado y las condiciones laborales del profesorado (Moreno, 2011, 37-40) áreas de oportunidad que representan el atender a los medios existentes, en la medida que se puede incidir.

El reto del trabajo en el aula para el desarrollo y aplicación de modelos y metodologías centradas en el estudiante son una necesidad para el trabajo profesional del docente universitario y brindan la oportunidad de atender la problemática que representa la comprensión y producción de textos, ya que como señala Claxton (citado por Martín, 2008, 73) "la primera función de la educación en un mundo incierto debería ser dotar a la juventud de la competencia y confianza en sí misma, necesarias para afrontar bien la incertidumbre: en otras palabras, ser buenos aprendices". Y en el caso de las competencias de comunicación con el conocimiento y uso del lenguaje, sin importar de qué nivel educativo se trate, reviste una vital importancia.

En este siglo XXI el discurso sobre aprender para la vida (Delors, 1996, 14) llevó a un sinnúmero de iniciativas y propuestas para mejorar las actividades didácticas que propiciaran el desarrollo de saberes, habilidades y actitudes desde la perspectiva del aprendizaje centrado en el estudiante; para la Universidad de Guadalajara se consolidó a través del Modelo Siglo 21 (Rectoría General, 2007) y desde entonces los programas educativos intentan trabajar con estas expectativas, para dar cumplimiento a los acuerdos establecidos por la UNESCO desde 1998.

Por ejemplo, el artículo primero de Misiones y funciones de la Educación Superior señala en su inciso b que la educación superior es un "espacio abierto... que propicia el aprendizaje permanente... con el fin de formar ciudadanos que participen activamente en su sociedad y estén abiertos al mundo...” (UNESCO, 1998, párr. 3) y es a través de sus habilidades de comunicación

Villafuerte Cosme doi: https://doi.org/10.36799/el.v2i1.50 Volumen 2, número 1, Año 2017, ISSN: 2448-5942 
que pueden lograr este objetivo. De ello se desprende el valor de Aprender a aprender: clave para el aprendizaje a lo largo de la vida (Martín, 2008, 72), lo cual implica desarrollar "capacidades que permiten al estudiante conocer y regular sus propios procesos de aprendizaje" (Martín, 2008, 73).

De acuerdo con la propuesta de la SEP y el Plan Nacional de Desarrollo en su apartado de Educación (Peña, 2013, 57-68), se trata de concatenar saberes y desde la educación básica se pretende la conquista de la lengua, como parte del perfil de egreso: "Utiliza el lenguaje materno, oral y escrito para comunicarse con claridad y fluidez, e interactuar en distintos contextos sociales y culturales..." (SEP, 2011), para unir con la educación media superior, donde se establece como competencia genérica en el apartado Se expresa y se comunica, que señala:

Escucha, interpreta y emite mensajes pertinentes en distintos contextos mediante la utilización de medios, códigos y herramientas apropiados.

- Expresa ideas y conceptos mediante representaciones lingüísticas, matemáticas o gráficas.

- Aplica distintas estrategias comunicativas según quienes sean sus interlocutores, el contexto en el que se encuentra y los objetivos que persigue.

- Identifica las ideas clave en un texto o discurso oral e infiere conclusiones a partir de ellas.

- Se comunica en una segunda lengua en situaciones cotidianas.

- Maneja las tecnologías de la información y la comunicación para obtener información y expresar ideas (Acuerdo 444 SNB-SEMS, 2008, 3).

Entonces, la educación superior supone que estas capacidades y destrezas han sido desarrolladas en la trayectoria que corresponde a los niveles básico y medio superior; sin embargo la evidencia entre los alumnos de licenciatura muestra serias debilidades y deficiencias, sobre los indicadores antes mencionados y en situaciones como la desmotivación hacia la lectura, el manejo de vocabulario, la comprensión y fluidez, entre otras dificultades. Esto estaría en consonancia con las estadísticas nacionales. Ante este panorama se propone un trabajo sobre las estrategias didácticas, con el objetivo de mejorar la adquisición de saberes teóricos y prácticos sobre la lengua y la literatura.

\section{EL DESARROLLO DE COMPETENCIAS EN LENGUA Y LITERATURA}

Villafuerte Cosme doi: https://doi.org/10.36799/el.v2i1.50 Volumen 2, número 1, Año 2017, ISSN: 2448-5942 
El sistema "competencias de lengua y literatura" tiene un referente multimodal e interactivo en su complejidad, ya que si se considera que "el concepto de competencia más generalizado y aceptado es el de 'saber hacer en contexto'. El 'saber hacer', lejos de entenderse como 'hacer' a secas requiere de conocimiento (teórico-práctico), afectividad, compromiso, cooperación y cumplimiento, lo cual se expresa en el desempeño” (Schmall y Ruíz-Tagle, 2008, 150). Por ello la selección de estrategias didácticas implica identificar en ellas la viabilidad y la posibilidad de integrar saberes, habilidades, valores y con ello desarrollar capacidades y adquirir nuevos conocimientos, que sean observables.

Si bien el perfil de egreso enuncia rasgos de competencia para las Letras Hispánicas en diferentes dimensiones, se hace necesario ir más allá y acotar las competencias que se espera desarrollar en las unidades de aprendizaje. Para el caso de diseñar proyectos que contribuyan al desarrollo de competencias de lectura y escritura, es necesario también identificar los atributos que se espera sean observables tras cursar por la trayectoria formativa. Entre ellos están la redacción de direfentes tipos de texto con correcta ortografía y sintaxis, también el manejo de diferentes tipos de texto para su análisis e interpretación y el uso de esta información para hacer propuestas e iniciativas de lingüística y literatura que incluyan el contacto con la tecnología digital.

No hay un acuerdo generalizado sobre el enfoque por competencias en la educación superior, la revisión de información muestra diversas maneras de hacer interpretaciones curriculares; desde los años 70 en que surge el enfoque en Estados Unidos, el paso del tiempo ha hecho migrar el enfoque de las exigencias empresariales para incrementar eficiencia y productividad, hasta la prospección de la sociedad que busca resultados de aprendizaje que se convierta en capital sociocultural, por lo que desarrollar competencias no sólo es aprender, aprender a aprender y aprender para la vida, sino mantener la autodirección y autoevaluación como guía de los propios avances (ANUIES, 1999; Delors, 1996);Deusto, 2015; Schmal y RuízTagle. 2007, 147-149:UNESCO, 2010).

Una de las autoras que en México ha dedicado sus esfuerzos a clarificar el concepto de competencias es Laura Frade (2009) que en su libro Desarrollo de Competencias en Educación: desde preescolar hasta bachillerato desarrolla cinco capítulos para revisar desde la necesidad de un profundo cambio educativo hasta las diferentes y novedosas necesidades de evaluación. Este antecedente de investigación recupera los conceptos teóricos de las competencias en el ámbito

Villafuerte Cosme doi: https://doi.org/10.36799/el.v2i1.50 Volumen 2, número 1, Año 2017, ISSN: 2448-5942 
educativo, el diseño curricular, las clases y las formas de evaluar, más allá de los exámenes, todos estos conceptos aplican perfectamente a las necesidades en el estado del arte de la educación superior.

La socioformación de competencias que propone Sergio Tobón (2012), quien señala que es necesario animar a la autonomía, no sólo del aprendizaje sino de la participación activa en el entorno que se desarrollan las comunidades educativas, precisa que el propósito de esta idea es "un aprendizaje integral, un proyecto ético de vida sólido y las competencias necesarias para afrontar los retos de los diversos contextos" (Tobón, 2012). Así propone un abordaje renovado del currículo que busca el desarrollo de las potencialidades de los estudiantes con base en prácticas educativas que generan emprendimiento creativo (Tobón, 2012), esta forma de acercarse a las competencias de manera transversal, será el insumo procedimental de esta investigación: las competencias se desarrollan de manera imbricada.

La Universidad de Deusto inició en el 2000 un proceso de transformación institucional que consiguió el establecimiento de treinta y cinco competencias clasificadas en tres categorías: instrumentales, interpersonales y sistémicas, sobre ellas se plantean niveles y atributos con indicadores que las describen para hacerlas observables, se "ponen en juego los diferentes tipos de destrezas y habilidades para convertirse en auténticas competencias cuando su dominio ha sido alcanzado... un aprendizaje integral que ayuda al alumno a desarrollarse como persona" (Villa y Poblete, 2007, 18). Con estaa visión es que se establece el valor del trabajo por proyectos.

\section{EL TRABAJO POR PROYECTOS}

El Aprendizaje Basado en Proyectos (ABP) es organizado para integrar teoría y práctica, se pretende potenciar habilidades intelectuales que superen la memorización y promuevan la responsabilidad personal y de equipo "al establecer metas propias, así como fomentar el pensamiento autocrítico y evaluativo" (Álvarez, Herrejón, Morelos y Rubio, 2010, 2).

Por lo tanto se establece que "en el aprendizaje por proyectos lo más importante no es el producto o resultado final sino el proceso de aprendizaje y profundización que llevan a cabo los estudiantes (Pereira Baz, 2014, 1-3. El origen de esta estrategia se remonta a principios del siglo XX. Cuando Kilpatrick de la Universidad de Columbia publicó su trabajo "Desarrollo de

Villafuerte Cosme doi: https://doi.org/10.36799/el.v2i1.50 Volumen 2, número 1, Año 2017, ISSN: 2448-5942 
Proyectos” en 1918 (ITESM,s.f., 2), donde se exponía las “características de la organización de un plan de estudios de nivel profesional basado en una visión global del conocimiento que abarcara el proceso completo del pensamiento, empezando con el esfuerzo de la idea inicial hasta la solución del problema” (ITESM,s.f., 2), fue el inicio de considerar que en la educación superior se debe favorecer la identificación de situaciones problemáticas sobre las que la intervención promueva soluciones.

"El desarrollo de proyectos, así como el desarrollo de solución de problemas, se derivaron de la filosofía pragmática que establece que los conceptos son entendidos a través de las consecuencias observables y que el aprendizaje implica el contacto directo con las cosas" (ITESM,s.f., 2), lo cual interpretado didácticamente invita a crear un sistema de intervención que a manera de secuencia didáctica utilice estas posibilidades para mejorar los aprendizajes.

Esta actividad didáctica aplica como método de enseñanza la premisa de que "los estudiantes lleven a cabo la realización de un proyecto en un tiempo determinado para resolver algún problema o abordar alguna tarea mediante la planeación, diseño y realización de una serie de actividades" (Tippelt y Lindemann citado en Manual de formación docente, 2009,16). Se fundamenta en el aprendizaje experiencial y reflexivo; en él toma importancia el proceso de investigar para generar y aplicar nuevos conocimientos.

"Todo proyecto, sea del tipo que sea, implica tres elementos: una propuesta de hacer o conocer algo, unos medios para llevarlo a cabo y un producto o realización que se puede evaluar y mejorar" (Galeano, 2010, 1). La ambigüedad de estos tres elementos da también la flexibilidad para aplicar en diferentes contextos, por lo que la libertad para hacer un proyecto, la capacidad para interconectar sus conocimientos y el uso de la información con la que cuentan, mas la investigación y el interés por una idea a desarrollar logra acciones que pueden ser evaluables y mejorables.

De tal manera que el seguimiento de esta estrategia empata con la idea de la multimodalidad y la importancia del cambio de pensamiento que propone Kress con respecto al lenguaje y que hace una transformación del diseño de nuevas teorias sobre los significados (Kress, 2000, 337-340), pero también sobre la interactividad, ya que siguiendo las fases de información, elaboración de un plan de trabajo, estructuración del procedimiento metodológico,

Villafuerte Cosme doi: https://doi.org/10.36799/el.v2i1.50 Volumen 2, número 1, Año 2017, ISSN: 2448-5942 
planeación de instrumentos y medios de trabajo y la realización (Manual de Formación Docente, 2009,16-17), genera un sinnúmero de posibilidades de acción para la comprensión y producción de textos.

Por lo que el trabajo por proyectos implica que desde el inicio de la idea aparezca la multimodalidad: el mismo enunciado de un pensamiento se transformará en un proyecto ejecutable y medible y la constante retroalimentación para avanzar en el diseño, las estrategias, los recursos y conocimientos necesarios, así como la integración de actividades y su modo de evaluar (tanto el proyecto como el proceso) hace una dinámica interactiva permanente, características que son deseables en el desarrollo de competencias de lengua y literatura.

\section{METODOLOGÍA}

A partir de una investigación cualitativa que guía el trabajo por proyectos en estas unidades de aprendizaje se sistematiza esta experiencia didáctica, en cuanto a que se trata de un proceso formativo que no espera calificar sino cualificar, por lo que los registros son a partir de diarios y bitácoras de clase realizados en las diferentes unidades de aprendizaje durante los diferentes semestres. Esto implicó que la retroalimentación de los resultados en cada periodo fuera modificando y reestructurando tanto el programa de estudios como el proceso dinámico del aula y el seguimiento de los proyectos de los alumnos.

Los estudiantes son guiados en el proceso que comienza con la investigación para argumentar la selección del proyecto:

$>$ Buscar, leer y comprender información hasta que se pueda aclarar un tema, cuando se logra clasificar la información de la primera búsqueda y selección de información las preguntas ya no son para conocer sino para plantear preguntas de investigación.

$>$ Entonces se puede definir y delimitar el tema o problema y se pueden plantear objetivos -que pueden modificarse, apenas son guía de una nueva revisión de literatura-

Villafuerte Cosme doi: https://doi.org/10.36799/el.v2i1.50 Volumen 2, número 1, Año 2017, ISSN: $2448-5942$ 
A partir de este momento la reflexión, análisis, interpretación y síntesis de lo conocido permite plantear lo desconocido: la investigación (y después un proyecto de gestión);

$>$ De este modo puede elegirse la pregunta que guiará el eje de investigación, se plantea el objetivo general y los particulares, si se requiere la hipótesis.

$>$ Es hasta que se revisan los objetivos que se puede elegir el método y definir la metodología de la investigación (y de la gestión) y ...

Se vuelve a revisar literatura, se eligen o diseñan técnicas e instrumentos

En este momento la competencia para investigar se centra en el conocimiento de un método que se aplica para lograr resultados y luego se utilizará para diseñar un proyecto lingüístico - literario.

$>$ A partir de este momento se está desarrollando la investigación hasta tener resultados y conclusiones que guiarán la gestión y

que deberán reportarse en un informe. (Villafuerte, 2016, 93-94)

Con este proceso se guía la construcción de un proyecto lingüístico y literario, que generalmente se convierte en un taller o club de lectura, donde proponen temas y títulos que no siempre pertenecen a la curricula que han cursado. Ese resultado es la evidencia de la multimodalidad e interactividad que señalan los logros sobre la comprensión de la literatura, la producción de textos, la interacción con estos como objetos de estudio, la consideración del contexto y sus saberes previos, así como el uso de la tecnología, ya que con esta metodología desde la misma redacción del proyecto se construye multimodalmente (temas de investigación, intereses, observación de campo, retroalimentación oral, trabajo de construcción sintáctica, etc.) y se genera interactividad tanto interpersonal como mediática, en la búsqueda y selección de información, la toma de decisiones para construir textos diversos y utilizar proyectos literarios holísticos, así como en los instrumentos pertinentes en el proyecto para darle seguimiento y verificar su éxito.

Villafuerte Cosme doi: https://doi.org/10.36799/el.v2i1.50 Volumen 2, número 1, Año 2017, ISSN: $2448-5942$ 
A partir del programa de estudios se diseñan las secuencias didácticas y siguiendo el proceso descrito para iniciar con una idea y concluir con un informe se estableció el proceso para realizar esta investigación:

\section{Primera etapa}

1.Identificación de la relación del programa con las características de los estudiantes para establecer el trabajo por proyectos.

Segunda etapa

2. Registro periódico de los avances en la bitácora de grupo y el diario de la observación docente, donde se describe como se transforman las ideas de los estudiantes y como re realizan los ajustes en la dinámica de clase para dar seguimiento a las ideas.

3. Realización de ejercicios de autoevaluación donde los estudiantes revisan su progreso en relación al contenido de la unidad de aprendizaje y el aprovechamiento del proyecto. Se establecen 3 mediciones (ciclos de 4 semanas) para verificar que los proyectos alcanzan su propósito.

4. Con esta información se estructura cada semestre el ajuste didáctico para desarrollar competencias de lengua y literatura.

5. Se utiliza el correo electrónico, la búsqueda de información en la web y diferentes soportes on line para retroalimentar las actividades o como propuesta de los proyectos.

\section{Tercera etapa}

6. Se realiza un análisis permanente de la relación del programa con los logros de los estudiantes y se mantiene coherencia entre saberes, habilidades, capacidades y valores.

8. La investigación continúa en proceso de mejora continua. Con el diseño y creación de actividades que favorezcan el logro de competencias de lectura y escritura a partir del trabajo por proyectos.

Villafuerte Cosme doi: https://doi.org/10.36799/el.v2i1.50 Volumen 2, número 1, Año 2017, ISSN: 2448-5942 


\section{¿CÓMO ABORDAMOS LA SITUACIÓN?}

A partir de la guía para la elaboración de una secuencia didáctica que propone Ángel Díaz Barriga (2013, 1-15) que señala que su estructura se integra con dos elementos que se realizan de manera paralela: la secuencia de actividades para el aprendizaje y la evaluación inscrita se genera la propuesta didáctica, que se transforma durante el desarrollo de los proyectos (no en la meta sino en las formas para lograrla), donde el contenido es la evidencia del aprendizaje y mantiene una retroalimentación permanente. Para esta propuesta se parte de tres ciclos de cuatro semanas para guiar el proceso de los proyectos. Es decir, a través de un semestre se pueden vivir tres oportunidades para incrementar las competencias.

Con la definición de la estrategia tenemos tres elementos: un tiempo determinado para resolver un problema o abordar una tarea; la planificación, diseño y realización de una serie de actividades y la aplicación de aprendizajes adquiridos y el uso efectivo de los recursos. En la fundamentación del aprendizaje orientado a proyectos se señala el valor que representa que los estudiantes asuman una mayor responsabilidad de su propio aprendizaje, lo que evita que sea fragmentado, así se vuelve centrado en lo que va a aprender (ITESM, 1999, 3) para utilizar de manera integral todos los recursos que el estudiante identifica y descubre -estos son los más enriquecedores-.

Si se consideran las seis fases que componen este método-técnica: informar, planificar, decidir, realizar, controlar, y valorar-reflexionar o evaluar (Tippelt y Lindemann, 2001, 6-8) se puede observar que su éxito depende de la guía para consolidar cada etapa en favor de resultados en el avance de conocimientos de los estudiantes, la guía de este proceso es el modelo de didáctica de la comprensión (Tuffanelli, 2010, 61) que señala la importancia de educar para comprender; lo cual implica contribuir para que el proceso enseñanza-aprendizaje se base en autoinstrucciones verbales que conduzcan a:

o Tener claro el objetivo con el que se lee o se escucha

o Generar preguntas durante una lectura (y durante el proceso)

o Distinguir las informaciones conocidas de las todavía desconocidas

o Identificar las palabras desconocidas

o Releer las partes que no estén claras (revisar la información)

o Aprender a construir imágenes mentales

Villafuerte Cosme doi: https://doi.org/10.36799/el.v2i1.50 Volumen 2, número 1, Año 2017, ISSN: 2448-5942 
Estos elementos señalan el acompañamiento que se realiza para que los alumnos construyan proyectos de lengua y literatura, diseñen ambientes de aprendizaje y lo complementen con la gestión en función de espacios reales para aplicar sus propuestas. La inclusión de la tecnología de acuerdo con Litwin (2005 en González López Ledesma, 2014, párr. 2) puede ser en cuatro esenarios educativos: "el de ayuda, el optimista, el de la producción y el de la problematización".

En el primero, el docente genera propuestas en las cuales la tecnología se presenta como una "herramienta" en un sentido artefactual y se emplea en diversas actividades como un objeto más, sin ser sometida a una integración en el marco de una propuesta didáctica y en función de un contenido. En el segundo prevalece el "encantamiento por la herramienta". La tecnología cobra un valor per se y un rol central más allá del contenido a ser abordado y el enfoque didáctico.

En el tercer escenario, a diferencia de los anteriores, el docente traslada su mirada a la relación entre el contenido y la tecnología, y centra la propuesta didáctica en el uso de la tecnología para producir "algo" con el contenido. El cuarto escenario es problematizador, en tanto que ahí la inclusión tecnológica se inscribe en la controversia: tecnología y contenido disciplinar se articulan y tensionan en una propuesta didáctica en la que ambas dimensiones son interdependientes. La tecnología colabora en la problematización del contenido y el contenido disciplinar se inscribe, necesariamente, en el acercamiento tecnológico propuesto (González López Ledesma, 2014, párr. 2-3).

Lo ideal es llegar al uso desde la problematización, sin embargo no es una constante en el resultado que se ha obtenido, el segundo escenario no puede ser la referencia de esta propuesta, ya que aun cuando la producción de textos y las lecturas se hacen on-line, el encantamiento por la herramienta estancaría el proceso del enfoque de proyectos. La meta es hacer uso de la tecnología para mejorar la posibilidad de leer, escribir, argumentar y construir nuevos conocimientos, lo cual implica alfabetizaciones múltiples (Manghi, 2012, 9) que consideren que hay nuevos formatos de presentación de información interactivos y constructivistas, que incluyen diagramas, fotografías, videos, formatos de páginas web, colores. Se requiere de reconocer el componente visual en los modelos de comprensión lectora.

El uso de la tecnología ha evolucionado hasta el e-learning para la formación profesional; herramientas tecnológicas y recursos didácticos van integrandose en entornos virtuales que

Villafuerte Cosme doi: https://doi.org/10.36799/el.v2i1.50 Volumen 2, número 1, Año 2017, ISSN: 2448-5942 
incluso llegan a la teleformación (García y Cabero, 2016, 168). Cada grupo de estudiantes tiene su propia dinámica, sin embargo, en cuanto al manejo de las tecnologías, su mínimo común es el uso del correo electrónico, telefonía celular y el conocimiento de búsqueda de información en internet, por lo que esto también se convierte en el mínimo de la multimodalidad y la interactividad.

El componente que mejor evidencía la apropiación de un proyecto por parte de los estudiantes es la elaboración de materiales didácticos para hacer funcionar sus propuestas, lo que incluye su cuidado y la integración de recursos tecnológicos, como la creación de un grupo de face book para integrar un club de lectura, en el que comparten los textos en formato de pdf y "suben" imágenes y caricaturas para comentar los textos propuestos. La revisión del impacto del uso de la tecnología para la apropiación de competencias de lengua y literatura es motivo de otro proyecto de investigación.

Por lo que respecta al ABP, en términos generales la estrategia tiene una primera etapa que es completamente guiada por el docente, la segunda es desarrollada por los alumnos con una propuesta que puede ser concensada o sugerida por el docente y una tercera etapa que es completamente autogestiva, donde el estudiante trabaja integrando los aprendizajes y descubrimientos de las primeras ocho semanas. Aunque de manera transversal se lleva un proceso imbricado que rompe las delimitaciones rígidas y le da flexibilidad para conseguir las competencias enunciadas en las unidades de aprendizaje.

Esto permite disminuir el impacto de los riesgos en el uso del trabajo por proyectos, como la dificultad de actuar con estudiantes poco motivados o con expectativas negativas en su rendimiento académico o la dificultad de aplicar el método con estudiantes que carezcan de conocimientos y experiencias relacionadas con los contenidos sobre los que se desea aplicar.

\section{RESULTADOS PRELIMINARES}

Durante los años en que se ha realizado este trabajo se ha ido mejorando el desempeño de esta propuesta, en su primera etapa los proyectos se enmarcaron en el conocer (la región y los espacios de desarrollo de la profesión); la segunda etapa logró una vinculación con el ayuntamiento formando promotores de lectura que llevaron clubes a las colonias y en espacios

Villafuerte Cosme doi: https://doi.org/10.36799/el.v2i1.50 Volumen 2, número 1, Año 2017, ISSN: 2448-5942 
de trabajo, tan diversos como un asilo o una panadería. En este momento se encuentra en una tercera etapa que tiene la aspiración de conducir a procesos formales de investigación, sin descartar la actividad social y sobre todo con la integración de las evidencias de profesionalización que manifiestan los alumnos. Es decir, las etapas se han marcado por los cambios en el uso del trabajo por proyectos (mejora docente y de los estudiantes).

Con la referencia de clasificación de competencias de la Universidad de Deusto (2015) se considera que esta estrategia desarrolla habilidades de investigación, trabajos autogestivos, por objetivos y desarrollo del espíritu emprendedor que se traducen en logros sobre la comprensión de la literatura y la producción de textos, vinculados con una interacción entre los objetos de estudio, su contexto y sus saberes previos, que integran el uso de las tecnologías.

Por ejemplo, con respecto al conocimiento de la literatura la diversidad de proyectos más reciente generó conocimiento dedicado a los autores jaliscienses; los heterónimos de Fernando Pessoa; la obra de José Revueltas; la relectura del libro publicado por José Vasconcelos con lecturas clásicas para niños. La producción de textos va desde los mensajes de correo electrónico, hasta la elaboración de guía de estudio y ensayos, además de crónicas sobre el desarrollo de los proyectos. Características que corresponden a la multimodalidad: conectada, hipertextual, compartida, colaborativa, cambiante, informal (Pujolà, 2011) que obliga a los profesores a ser profesores de comunicación lingüística y digital.

El análisis particular del uso de las tecnologías es relevante, sin embargo, como el objetivo de este artículo es enfatizar que el trabajo por proyectos desarrolla competencias de lengua y literatura, la multimodalidad tecnológica sólo está referida, ya que el interés es señalar que la interactividad en la Era Digital se sujeta al conjunto de beneficios de conocer más allá de la tecnología, que tiene principios humanistas, pero sobre todo en la integración de los objetos de estudio, su contexto y sus saberes previos.

\section{LA DISCUSIÓN}

Los datos para hacer la discusión que se sistematizan en esta experiencia didáctica se obtuvieron de diarios de observación de la práctica docente, bitácoras de clase, observación participante, ejercicios de autoevaluación orales y escritos, así como sesiones plenarias para

Villafuerte Cosme doi: https://doi.org/10.36799/el.v2i1.50 Volumen 2, número 1, Año 2017, ISSN: 2448-5942 
compartir la experiencia de la realización de proyectos. El procesamiento de la información se efectuó a través del análisis de contenido con la selección de categorías: como las competencias genéricas, la identificación de conocimientos, habilidades y valores, así como sobre los temas, ideas y propuestas que los diferentes grupos fueron realizando. De esta manera se cumple la propuesta de Rossell y Más (2003) de sistematizar a partir de la elaboración de proyectos y su proceso desde el inicio hasta la transformación final en cada ciclo escolar.

Los elementos identificados para el desarrollo de habilidades en la lectoescritura universitaria, muestran que usar proyectos es una estrategia favorable, pues promueve la autonomía en ejercicios de redacción, creación de textos, su análisis e interpretación, así como la elección de los medios más convenientes para su comunicación. Como estrategia, la principal adaptación es hacer que los ciclos puedan cumplirse en un semestre y que los estudiantes se hagan conscientes de su desarrollo antes, durante y después de vivir la experiencia formativa. El obstáculo fundamental es el tiempo y la multiplicidad de actividades a las que deben responder los alumnos, ya que eso dificulta las etapas y su evaluación progresiva. Para mejorar los resultados el docente es un agente dinamizador insustituible, ya que propone, motiva, celebra y reorienta los esfuerzos para alcanzar los propósitos establecidos.

Por otra parte, el conocimiento de los indicadores enunciados en las competencias que se forman desde la educación básica, permite canalizar los esfuerzos y sobre todo propiciar la autogestión de saberes y habilidades que los jóvenes -ya como adultos responsables de sí mismos- deben hacer para lograr ser competentes como alumnos universitarios. Este diálogo es la oportunidad real de trabajar centrados en el estudiante y su aprendizaje.

Los trabajos por proyectos desarrollan un proceso imbricado de competencias porque son un proceso permanente de reflexión y toma de decisiones con base en objetivos definidos previamente por cada interesado en su propia idea. Contribuyen en la formación de competencias de lengua y literatura ya que la dinámica implica un compromiso con el conocimiento, las acciones y la evaluación, lo que mejora los ciclos del proceso de aprendizaje representa una responsabilidad total con lo que se desea aprender.

Una ventaja de este modo de desarrollar actividades de lectura y escritura es que los estudiantes se hacen conscientes de sus debilidades y deficiencias, convirtiéndolas en áreas de

Villafuerte Cosme doi: https://doi.org/10.36799/el.v2i1.50 Volumen 2, número 1, Año 2017, ISSN: 2448-5942 
oportunidad, lo cual permite que se desarrollen habilidades de investigación, autogestión, trabajo por proyectos e iniciativa, componentes de las competencias necesarias como egresado de la educación superior. En este punto se puede hacer evidente que todo texto va más allá del mensaje escrito; que hay diferentes maneras de interacción y comunicación que se complementan y se hacen complejas; que los diferentes niveles de interpretación se dan en los diferentes modos y sistemas semióticos.

La elaboración y comprensión de textos desde el enfoque didáctico centrado en el estudiante, rescata los principios que convierten a este proceso en un acto con objetivos que se logran escribiendo (Cassany, 1999, 15-17), una competencia que en la era 2.0 requiere de no perder de vista que el concepto on-line no elimina la interacción personal y sobre todo el valor creativo de generar proyectos y ejecutarlos para participar del espacio social donde se desenvuelven docentes y estudiantes: las comunidades a las que pertenecemos, donde nuestro enfoque sobre la didáctica favorece o entorpece la lectoescritura.

El entramado es complejo e imbricado, pero la satisfacción de compartir el aprendizaje vale la pena, sobre todo porque se trata de una integración real de saberes que humaniza el proceso de lectoescritura y compromete la comprensión y el uso de textos literarios y de corte académico, pero también centra el interés en la pasión por ser y aprender desde la perspectiva del estudiante, lo cual cumple la función integradora, ya que el conocimiento fragmentado limita las posibilidades creativas y de contextualización.

Un proyecto con estas características -de investigación previa, detección de intereses personales y su contextualización y de largo aliento- promueve ese aprendizaje para la vida que se propone la educación actual. Esta experiencia ha posibilitado comprender la importancia de los diferentes estilos para redactar, desde un cartel de invitación a una lectura en voz alta, hasta la selección de lecturas incluyendo el tamaño de la letra y la distriución de los párrafos, también ha invitado a los participantes a conocer más de los géneros literarios -e incluso periodísticos-, por ejemplo con la propuesta "lectores al rescate" que generó una sección en la gaceta universitaria para promover los libros de la biblioteca.

En cuanto al proceso de la investigación, la primera etapa como docente implicó sensibilidad para detonar el interés de los estudiantes, ya que la planeación del programa no

Villafuerte Cosme doi: https://doi.org/10.36799/el.v2i1.50 Volumen 2, número 1, Año 2017, ISSN: $2448-5942$ 
implica dificultad, su relación con la estrategia didáctica se mejora con el uso de la misma, pero los intereses de los estudiantes son cambiantes. La segunda etapa implica disciplina para mantener el registro, que a veces es extenso y otras de solo unas notas, la bitácora de grupo es muy útil para verificar el seguimiento de lo que sucede en el aula y fuera de ella; el diario de observaciones de la práctica docente es el hábito que permite la mejora continua.

Por lo que respecta a los ejercicios de autoevaluación, las preguntas centrales fueron ¿cuál es tu proyecto?, ¿cuáles son los recursos que se requieren?, ¿cómo se va a ejecutar?, ¿cómo se va a medir? Las respuestas se modifican durante las tres veces que se efectúan. También se pregunta sobre los conocimientos de la materia y en la sesión final se contrasta la información. El análisis de esta información modifica las actividades que se realizan cada semestre. El uso de las tecnologías por parte del docente se centran en el correo electrónico, la búsqueda de información en la web y la plataforma moodle para los registros; los alumnos usan diferentes soportes on line para retroalimentar las actividades o como propuesta de los proyectos (WIX, Face book, cursos MOOC).

La tercera etapa consiste en un análisis permanente de los resultados de la estrategia, la relación del programa con los logros de los estudiantes y la coherencia entre saberes, habilidades, capacidades y valores. Hasta este momento la experiencia más significativa ha sido una trayectoria de proyecto que no solo sirvió para las unidades de aprendizaje señaladas, sino que se utilizó para otras materias, el servicio social, las prácticas profesionales y la titulación con un manual de actividades de comprensión lectora para alumnos de cuarto grado de primaria sobre autores jaliscienses. Empezó como una tarea de semestre y mantuvo la atención de la estudiante durante tres años. Al egresar inició su propia empresa para asesorar en habilidades de lecto escritura a alumnos de primaria.

Por estos resultados puede mantenerse la premisa de utilizar el trabajo por proyectos para integrar saberes y promover la multimodalidad como una actividad consciente y promotora de mejoras en las capacidades de lectura y escritura, tanto para los estudiantes, como para los docentes y por supuesto también en sus comunidades, ya que se convierten en agentes alfabetizadores funcionales, comprometidos desde su libertad y con un verdadero humanismo.

Villafuerte Cosme doi: https://doi.org/10.36799/el.v2i1.50 Volumen 2, número 1, Año 2017, ISSN: 2448-5942 
Finalmente, esta estrategia didáctica hace que los propios alumnos construyan sus objetos de estudio en el contexto del uso de las TIC's, utilizando sus saberes previos y redefiniendo la trayectoria en su educación profesionalizante. Como diría Daniel Cassany "Desde aprender cualquier oficio hasta cumplir con los deberes fiscales o participar en la vida cívica de la comunidad, cualquier hecho requiere cumplimentar impresos, enviar solicitudes, plasmar la opinión por escrito o elaborar un informe. Todavía más: el trabajo de muchas personas (maestros, periodistas, funcionarios, economistas, abogados, etc.) gira totalmente o en parte en torno a documentación escrita" $(1996,13)$.

Por lo tanto desarrollar competencias de lengua y literatura en el siglo XXI requiere conocimientos que dinamicen el aprendizaje. El trabajo por proyectos puede organizar estos elementos y sus necesidades para hacer procesos formativos integrales y de mejora continua que trasciendan en la profesionalización del estudiante de educación superior. Obteniendo productos observables que sean medibles con criterios de desempeño generados del propio interés del estudiante y la guía de su formación orientada por el docente.

\section{REFERENCIAS}

Acuerdo 444 SNB-SEMS. Diario Oficial de la Federación. 21 de octubre de 2008. 26 de enero de 2016.

<www.sems.gob.mx/work/.../1/.../Acuerdo_444_marco_curricular_comun_SNB.pdf>.

Álvarez, Valeria; Herrejón, Verónica del Carmen; Morelos, Mónica y María Teresa Rubio. “Trabajo por proyectos: aprendizaje con sentido". Revista Iberoamericana de Educación (2010): 1-13. rieoei.org/deloslectores/3202Morelos.pdf.

ANUIES. La educación superior en el siglo XXI. 1999.

<http://publicaciones.anuies.mx/pdfs/revista/Revista113_S5A2ES.pdf>.

Bertalanffy, Ludwig Von. Teoría General de los sistemas . México: FCE, 2006.

Bravo, Néstor. Competencias Proyecto Tuning-Europa, Tuning.-America Latina. Universidad de Chile, 2006.

Cassany, Daniel. La cocina de la escritura. Barcelona: Anagrama, 2004.

Villafuerte Cosme doi: https://doi.org/10.36799/el.v2i1.50 Volumen 2, número 1, Año 2017, ISSN: 2448-5942 
Cassany, Daniel. "Una declaración de principios". Construir la escritura. Barcelona: Paidós, 1999. $15-17$.

CIEP. Coordinación de Innovación Educativa y Pregrado. 2015. < http://ciep.cga.udg.mx/objetivos>.

Cortés Agustín. "La competencia en la educación”. complilación del IPESUJ. Ser alumno e doctorado en competencias educativas. Guadalajara: IPESUJ, 2013. 243.

CUSUR. "Licenciatura en Letras Hispánicas". 2008. Mapa curricular. 12 de mayo de 2010. <http://www.cusur.udg.mx/es/letras-hispanicas/mapa-curricular>.

Delors, Jacques. La educación encierra un tesoro. 1996.

<http://unesdoc.unesco.org/images/0010/001095/109590so.pdf>.

Deusto. Deusto Bussines School. 2015.

<http://www.dbs.deusto.es/cs/Satellite?blobcol=urldata\&blobheader=application $\% 2$

Fpdf\&blobheadername1 $=$ Expires\&blobheadername2 $=$ content -

type\&blobheadername3 $=$ MDT -

Type\&blobheadername4=Contentdisposition\&blobheadervalue $1=\mathrm{Thu} \% 2 \mathrm{C}+10+\mathrm{Dec}$ $+2020+16 \% 3 \mathrm{~A} 00 \% 3 \mathrm{~A} 00+$ GMT\&blobh>.

Díaz Barriga, Ángel. «Guía para la elaboración de una secuencia didáctica.» Comunidad de conocimiento. UNAM 2013: 1-15.

Didrikson, Axel. La Universidad del futuro. Relaciones entre la educación superior, la ciencia y la tecnología. México: Plaza y Valdez/UNAM, 2000.

Flores, José Humberto. Complejidad y Educación. 2010.

<http://rd.udb.edu.sv:8080/jspui/bitstream/11715/431/1/2.\%20Complejidad $\% 20 y \%$ 20educacion.pdf $>$.

Frade, Laura. Desarrollo de Competencias en Educación: desde preescolar hasta bachillerato. México: Inteligencia Educativa, 2009.

Galeano, María del Carmen. Trabajo por proyectos, una forma práctica de enseñar. 2010.

Villafuerte Cosme doi: https://doi.org/10.36799/el.v2i1.50 Volumen 2, número 1, Año 2017, ISSN: $2448-5942$ 
$<$ www.sociedadelainformacion.com>.

García Marcos; Cristian, Jorge y Julio Cabero Almenara. «Evolución y estado actual del e-learning en la Formación Profesional Española. ». 2016. RIED.

http://dx.doi.org/ried.19.2.15800

García Muriel, Loreto. La comunicación una experiencia de vida: manual de trabajo en grupos. México: Plaza y Valdez, 1996. 15.

Gil, Álvaro. Los retos del nuevo milenio: educar en las competencias para el contexto de la mundialización. s.f. <file:///C:/Users/N\%C3\%A9lida/Downloads/Dialnet-LosRetosDelNuevoMilenio$4897911 \% 20(1) \cdot p d f>$.

Gómez, Gonzalo. La teoría general de sistemas aplicada al análisis del centro escolar.

<http://www.mecd.gob.es/dctm/revista-de-

educacion/articulosre266/re2660113057.pdf?documentId=0901e72b813ce55f > .

González, Margarita e Ignacio Ramírez. Laformación de competencias profesionales: un reto en los proyectos curriculares universitarios. enero-junio de 2011. <http://www.odiseo.com.mx/2011/816/pdf/gonzalez-ramirez-formacion-competencias.pdf $>$.

González López Ledesma, Alejo Ezequiel. "Apertura. Revista de Innovación educativa”. Tipología de propuestas de abordaje de contenidos de Lengua y Literatura con inclusión de TIC.Octubre de 2014.

<http://www.udgvirtual.udg.mx/apertura/index.php/apertura/article/view/531/367>.

Herrán de la, Agustín. Didáctica Universitaria: La Cara Dura de la Universidad. S.f.

<https://www.uam.es/personal_pdi/fprofesorado/agustind/textos/caradura.pdf $>$.

ITESM. El método de proyectos como técnica didáctica. s.f. 12 de marzo de 2016.

<http://sitios.itesm.mx/va/dide2/tecnicas_didacticas/aop/proyectos.pdf > .

Kress, Gunther. Multimodality: Challenges to Thinking About Language. TESOL. Quarterly 34.2 (2000): 337-340.

Villafuerte Cosme doi: https://doi.org/10.36799/el.v2i1.50 Volumen 2, número 1, Año 2017, ISSN: 2448-5942 
<http://www.jstor.org.wdg.biblio.udg.mx:2048/stable/3587959?seq=2\#page_scan_ta b_contents>.

."Reading Images: Multimodality, representation and New Media". Communication \& Mass Media Complete (2016): 110-119.

Latorre, Víctor. “Carreras basadas en competencias”.s.f. Proyecto Tuning - América Latina. <file:///C:/Users/N\%C3\%A9lida/Downloads/TUNING_AL_Victor_Latorre_texto .pdf $>$.

López de la Madrid, María Cristina. "Uso de las TIC en la educación superior de México. Un estudio de caso”. Apertura 7.7 (2007): 63-81. junio de 24 de 2009.

Manghi, Dominique. "Diálogos Educativos". enero de 2012. La perspectiva multimodal sobre la comunicación. Desafíos y aportes para la enseñanza en el aula . 11 de septiembre de 2015. <http://www.dialogoseducativos.cl/revistas/n22/manghi $>$.

Sin autor. Manual de Formación Docente. Guadalajara: UdG, 2009.

Martín Ortega , Elena. Aprender a aprender: clave para el aprendiaje a lo largo de la vida. noviembre de 2008. Tribuna Abierta. <www.mecd.gob.es/revista-cee/pdf/n9-martin-ortega.pdf>.

Mir, Anna. Las competencias transversales en la Universidad Pompeu Fabra. 2007. $<$ http://www.redu.um.es/Red_U/m1 $>$.

Moncada, Jesús Salvador. Modelo educativo basado en competencias. México: Trillas, 2011.

Moreno Olivos, Tiburcio. "Didáctica de la Eduación Superior: nuevos desafíos en el siglo XXI". Perspectiva Educacional 50.2 (2011): 26-64. 18 de agosto de 2015. <www.perspectivaeducacional.cl > Inicio > Vol 50, No 2 (2011) > Moreno Olivos>. . "Didáctica de la educación superior nuevos desafíos en el siglo XXI". Perspectiva Educacional (s.f.): 26-54.

Nerici, Imídeo. Hacia una didáctica general. Buenos Aires: Kapeluz, 1973. 
Peña, Enrique. Plan Nacional de Desarrollo 2013-2018. 2013. Gobierno de la República.

<http://pnd.gob.mx/>.

Programa de Formación de Académicos. "Cómo aprovechar al máximo las dos horas de clase”. 2012. Ibero Ciudad de México.

<http://www.ibero.mx/formaciondeprofesores/Apoyos $\% 20$ generales/wp $\% 20 \mathrm{AD} \% 2$ 0Comoaprovechar.pdf $>$.

Pereira Baz, Miguel Ángel. Ocho claves del aprendizaje por proyectos (2014).

<http://www.cedec.educalab.es/es/noticias-de.../1559-8-claves-del-aprendizaje-porproyectos $>$.

Pujolà, Juan-Tomàs. "Discurso 2.0 enseñar a hilar textos multimodales". TodoELE2.0 (2011). $<$ http://todoele.org/todoele20/content/discurso-20-ense $\%$ C3\%B1ar-hilar-textosmultimodales $>$.

Rectoría General. Modelo Educativo siglo 21. Guadalajara: UdG, 2007.

Rodríguez, Hernán. El paradigma de las competencias hacia la educación superior. junio de 2007. <http://www.redalyc.org/pdf/909/90915108.pdf>.

Rodriguez, María Luisa. "Aplicación de la teoria general de sistemas a la orientacion educativa y a la formacion de orientadores". s.f. El desarrollo interdisciplinar de la Teoria General de Sistemas.

<http://www.raco.cat/index.php/EducacioCultura/article/viewFile/70012/86409>.

Rosell, Washington y Martha Más. "El enfoque sistémico en el contenido de la enseñanza”. 2003. Instituto Superior de Ciencias Médicas de La Habana Facultad de Ciencias Médicas"Dr. Enrique Cabrera".

<http://www.bvs.sld.cu/revistas/ems/vol17_2_03/ems02203.htm>.

Schmall, Rodolfo y Andrés Ruíz-Tagle. "Una metodología para el diseño de un curriculum orientado a las competencias". Ingeniare. Revista chilena de ingeniería (2008): 147-158. www.redalyc.org/articulo.oa?id=77216204.

Villafuerte Cosme doi: https://doi.org/10.36799/el.v2i1.50 Volumen 2, número 1, Año 2017, ISSN: 2448-5942 
SEP. "Eduación básica”. 2011. 18 de febrero de 2016.

<http://www.curriculobasica.sep.gob.mx/index.php/plan-estudios/planestudios/perfil-egreso>.

Tippelt, Rudolf y Hans Lindemann. Septiembre de 2011. El Método de Proyectos. <www.uaa.mx/direcciones/dgdp/defaa/descargas/el_metodo_de_proyectos.pdf>.

Tobón, Sergio de Jesús. El currículo por competencias desde la socioformación. México: Limusa, 2012.

Tuffanelli, Luigi. Comprender ¿qué es? ¿cómo funciona? Madrid: Narcea, 2010.

Tuning. Alfa Tuning América Latina. 2007.

<file:///C:/Users/N\%C3\%A9lida/Downloads/LIBRO_TUNING_AMERICA_LA TINA_version_final_espanol\%20(1).pdf $>$. .Tuning América Latina. 2013. <http:/ /www.tuningal.org/>.

UNESCO. 9 de octubre de 1998. Declaracion mundial sobre la educacion superior. 14 de julio de 2016. $<$ www.unesco.org/education/educprog/wche/declaration_spa.htm>. “Conferencia Mundial sobre la educación superior 2009. La nueva dinámica de la educación superior y la investigación para el cambio social y el desarrollo”. 2010. Comunicado. <http://unesdoc.unesco.org/images/0018/001832/183277s.pdf>. .Replantear la educación. 2015.

<http://unesdoc.unesco.org/images/0023/002326/232697s.pdf>.

Valenzuela, Álvaro. Teoría y aplicaciones prácticas para estudiantes de pedagogía y profesionales de la educación. 2004. Enfoque de sistemas aplicado a la educación.

<http://www.alvarovalenzuela.cl/ENFOQUE.pdf>.

Villa, Aurelio. y Olga Villa. "El aprendizaje basado en competencias y el desarrollo de la dimensión social en las universidades". Educar (2007): pp. 15-48.

Villafuerte, N. "3.1 El enfoque sistémico". El negocio de la prensa local. Periódico el juglar, un estudio de caso en ciudad guzmán Jalisco. Cd. Guzmán: Tesis de grado (maestría) CUSUR, Diciembre de 2011.

Villafuerte Cosme doi: https://doi.org/10.36799/el.v2i1.50 Volumen 2, número 1, Año 2017, ISSN: $2448-5942$ 
Análisis e interpretación de documentos científicos como factor para desarrollar competencias de investigación en educación superior. Ciudad Guzmán: Tesis del doctorado en desarrollo de competencias educativas IPESUJ, 2016.

Villafuerte Cosme doi: https://doi.org/10.36799/el.v2i1.50 Volumen 2, número 1, Año 2017, ISSN: 2448-5942 